\title{
Integral Inequalities of Hermite-Hadamard Type for m-AH Convex Functions
}

\author{
Tian-Yu Zhang ${ }^{1, *}$, Feng $\mathbf{Q i}^{2,3}$ \\ ${ }^{1}$ College of Mathematics, Inner Mongolia University for Nationalities, Tongliao City, Inner Mongolia Autonomous Region, China \\ ${ }^{2}$ Department of Mathematics, School of Science, Tianjin Polytechnic University, Tianjin City, China \\ ${ }^{3}$ Institute of Mathematics, Henan Polytechnic University, jiaozuo City, Henan Province, China \\ *Corresponding author: zhangtianyu7010@126.com
}

Received April 09, 2014; Revised May 16, 2014; Accepted June 03, 2014

\begin{abstract}
In the paper, the authors introduce the concept "m-AH convex functions" and establish some inequalities of Hermite-Hadamard type for m-AH convex functions.
\end{abstract}

Keywords: Hermite-Hadamard's inequality, m-AH convex function, Hölder's inequality

Cite This Article: Tian-Yu Zhang, and Feng Qi, "Integral Inequalities of Hermite-Hadamard Type for m-AH Convex Functions." Turkish Journal of Analysis and Number Theory, vol. 2, no. 3 (2014): 60-64. doi: 10.12691/tjant-2-3-1.

\section{Introduction}

Throughout this paper, we use the following notations: $\mathbb{R}=(-\infty,+\infty), \mathbb{R}_{0}=[0,+\infty), \mathbb{R}_{+}=(0,+\infty)$.

We first recall several definitions.

Definition 1.1. A function $f: I \subset \mathbb{R} \rightarrow \mathbb{R}$ is said to be convex if

$$
f(t x+(1-t) y) \leq t f(x)+(1-t) f(y)
$$

holds for all $x, y \in I$ and $t \in[0,1]$.

Definition 1.2. A function $f: I \subset \mathbb{R}_{0} \rightarrow \mathbb{R}_{+}$is said to be geometrically convex if

$$
f\left(x^{t} y^{(1-t)}\right) \leq[f(x)]^{t}[f(y)]^{(1-t)}
$$

holds for all $x, y \in I$ and $t \in[0,1]$.

Definition 1.3 ([3]). A function $f:[0, b] \rightarrow \mathbb{R}$ is said to be $m$-convex if

$$
f(t x+m(1-t) y) \leq t f(x)+m(1-t) f(y)
$$

holds for all $x, y \in[0, b], t \in[0,1]$, and some $m \in(0,1]$.

Definition 1.4 ([11]). Let $f:[0, b] \rightarrow \mathbb{R}_{+}$be a positive function on $[0, b]$ and $m \in(0,1]$. If

$$
f\left(x^{t} y^{m(1-t)}\right) \leq[f(x)]^{t}[f(y)]^{m(1-t)}(1.4)
$$

holds for all $x, y \in[0, b], t \in[0,1]$, then we say that the function $f(x)$ is $m$-geometrically convex on $[0, b]$.

Definition 1.5 ([12]). A function $f:[0, b] \rightarrow \mathbb{R}$ is said to be $(\alpha, m)$-convex where $(\alpha, m) \in[0,1]^{2}$, if we have

$$
f(t x+m(1-t) y) \leq t^{\alpha} f(x)+m\left(1-t^{\alpha}\right) f(y)
$$

for all $x, y \in[0, b], t \in[0,1]$.

We now recall some inequalities of Hermite- Hadamard type.

Theorem1.1 ([[1]. Theorem 2.2]). Let $f: I^{\circ} \subset \mathbb{R} \rightarrow \mathbb{R}$ be a differentiable mapping on $I^{\circ}$ and $a, b \in I^{\circ}$ with $a<b$. (i) If $\left|f^{\prime}(x)\right|$ is convex on $[a, b]$, then

$\left|\frac{f(a)+f(b)}{2}-\frac{1}{b-a} \int_{a}^{b} f(x) \mathrm{d} x\right| \leq \frac{(b-a)\left(\left|f^{\prime}(a)\right|+\left|f^{\prime}(b)\right|\right)}{8}(1.6)$

(ii) If $\left|f^{\prime}(x)\right|^{p /(p-1)}$ is convex on $[a, b]$ for $p>1$, then

$$
\begin{aligned}
& \left|\frac{f(a)+f(b)}{2}-\frac{1}{b-a} \int_{a}^{b} f(x) \mathrm{d} x\right| \\
& \leq \frac{b-a}{2(p+1)^{1 / p}}\left(\frac{\left|f^{\prime}(a)\right|^{p /(p-1)}+\left|f^{\prime}(b)\right|^{p /(p-1)}}{2}\right)^{(p-1) / p} .
\end{aligned}
$$

Theorem 1.2 ([[2], Theorems 2.3 and 2.4]). Let $f: I \subset \mathbb{R} \rightarrow \mathbb{R}$ be differentiable on $I^{\circ}$ and $a, b \in I^{\circ}$ with $a<b$ If $\left|f^{\prime}(x)\right|^{p}$ is a convex function on $[a, b]$ for $p>1$, then

$$
\begin{gathered}
\left|f\left(\frac{a+b}{2}\right)-\frac{1}{b-a} \int_{a}^{b} f(x) \mathrm{d} x\right| \\
\leq \frac{b-a}{4}\left(\frac{4}{p+1}\right)^{1 / p}\left(\left|f^{\prime}(a)\right|+\left|f^{\prime}(b)\right|\right) . \\
\left|f\left(\frac{a+b}{2}\right)-\frac{1}{b-a} \int_{a}^{b} f(x) \mathrm{d} x\right| \leq \frac{b-a}{16}\left(\frac{4}{p+1}\right)^{1 / p} \\
\times\left[\left(\left|f^{\prime}(a)\right|^{p /(p-1)}+3\left|f^{\prime}(b)\right|^{p /(p-1)}\right)^{(p-1) / p}\right. \\
\left.+\left(3\left|f^{\prime}(a)\right|^{p /(p-1)}+\left|f^{\prime}(b)\right|^{p /(p-1)}\right)^{(p-1) / p}\right] .
\end{gathered}
$$

Theorem 1.3 ([[4], Theorem 2]). Let $f: \mathbb{R}_{0} \rightarrow \mathbb{R}$ be $m$ convex and $m \in(0,1]$. If $f \in L([a, b])$ for $0 \leq a<b<\infty$, then

$$
\begin{aligned}
& \frac{1}{b-a} \int_{a}^{b} f(x) \mathrm{d} x \\
& \leq \min \left\{\frac{f(a)+m f(b / m)}{2}, \frac{m f(a / m)+f(b)}{2}\right\} .
\end{aligned}
$$


Theorem 1.4. ([[6], Theorem 2.2]). Let $I \subset \mathbb{R}_{0}$ be an open real interval and let $f: I \rightarrow \mathbb{R}$ be a differentiable function on $I$ such that $f^{\prime} \in L([a, b])$ for $0 \leq a<b<\infty$. If $\left|f^{\prime}(x)\right|^{q}$ is $m$-convex on $[a, b]$ for some given numbers $m \in(0,1]$ and $q \geq 1$, then

$$
\begin{aligned}
& \left|f\left(\frac{a+b}{2}\right)-\frac{1}{b-a} \int_{a}^{b} f(x) \mathrm{d} x\right| \\
& \leq \frac{b-a}{4} \min \left\{\left(\frac{\left|f^{\prime}(a)\right|^{q}+m\left|f^{\prime}(b / m)\right|^{q}}{2}\right)^{1 / q},\right. \\
& \left.\left(\frac{m\left|f^{\prime}(a / m)\right|^{q}+\left|f^{\prime}(b)\right|^{q}}{2}\right)^{1 / q}\right\} .
\end{aligned}
$$

In this paper, we will introduce the concept " $m-\mathrm{AH}$ convex functions" and establish some inequalities of Hermite-Hadamard type for $m$-AH convex functions.

\section{Definition and lemmas}

The concept of $m-\mathrm{AH}$ convex function may be introduced as follows.

Definition 2.1. A function $f: I \subset \mathbb{R} \rightarrow \mathbb{R}_{0}$ is said to be AH convex if for all $x, y \in I$ and $t \in[0,1]$ the inequality

$$
f(t x+(1-t) y) \leq\left[t(f(x))^{-1}+(1-t)(f(y))^{-1}\right]^{-1}
$$

holds. If the inequality (2.1) is reversed then $f(x)$ is said to be $\mathrm{AH}$ concave function.

Definition 2.2. A function $f:[0, b] \rightarrow \mathbb{R}_{0}$ is said to be $m$-AH convex for some given number $m \in(0,1]$, if the inequality

$$
f(t x+m(1-t) y) \leq\left[t(f(x))^{-1}+m(1-t)(f(y))^{-1}\right]^{-1}
$$

holds for all $x, y \in[0, b]$ and $t \in[0,1]$. if the inequality (2.2) reverses, then $f(x)$ is said to be $m$-AH concave.

When $m=1$, the $m-\mathrm{AH}$ convex function is AH convex function on $[0, b]$.

In order to establish some inequalities of HermiteHadamard type for $m$-AH convex functions, we find the following lemmas.

Lemma 2.1. Let $f: I \subset \mathbb{R} \rightarrow \mathbb{R}$ be differentiable on $I^{\circ}$, $a, b \in I^{\circ}$ with $a<b$, and $f^{\prime} \in L([a, b])$. Then

$$
\begin{aligned}
& f(x)-\frac{1}{b-a} \int_{a}^{b} f(u) \mathrm{d} u \\
& =\frac{(x-a)^{2}}{4(b-a)}\left(\int_{0}^{1} t f^{\prime}\left(t \frac{a+x}{2}+(1-t) a\right) \mathrm{d} t\right. \\
& \left.+\int_{0}^{1}(1+t) f^{\prime}\left(t x+(1-t) \frac{a+x}{2}\right) \mathrm{d} t\right) \\
& -\frac{(b-x)^{2}}{4(b-a)}\left(\int_{0}^{1}(2-t) f^{\prime}\left(t \frac{b+x}{2}+(1-t) x\right) \mathrm{d} t\right. \\
& \left.+\int_{0}^{1}(1-t) f^{\prime}\left(t b+(1-t) \frac{b+x}{2}\right) \mathrm{d} t\right) .
\end{aligned}
$$

Proof. Integrating by part gives

$$
\begin{aligned}
& \int_{0}^{1} t f^{\prime}\left(t \frac{a+x}{2}+(1-t) a\right) \mathrm{d} t \\
= & \frac{2}{x-a} \int_{0}^{1} t \mathrm{~d} f\left(t \frac{a+x}{2}+(1-t) a\right) \\
= & \frac{2}{x-a}\left(f\left(\frac{a+x}{2}\right)-\frac{2}{x-a} \int_{a}^{\frac{a+x}{2}} f(u) \mathrm{d} u\right) .
\end{aligned}
$$

Similarly, we have

$$
\begin{aligned}
& \int_{0}^{1}(1+t) f^{\prime}\left(t x+(1-t) \frac{a+x}{2}\right) \mathrm{d} t \\
& =\frac{2}{x-a}\left(2 f(x)-f\left(\frac{a+x}{2}\right)-\frac{2}{x-a} \int_{\frac{a+x}{2}}^{x} f(u) \mathrm{d} u\right), \\
& \int_{0}^{1}(2-t) f^{\prime}\left(t \frac{b+x}{2}+(1-t) x\right) \mathrm{d} t \\
& =\frac{2}{b-x}\left(f\left(\frac{b+x}{2}\right)-2 f(x)+\frac{2}{b-x} \int_{x}^{\frac{b+x}{2}} f(u) \mathrm{d} u\right), \\
& \quad \int_{0}^{1}(1-t) f^{\prime}\left(t b+(1-t) \frac{b+x}{2}\right) \mathrm{d} t \\
& =\frac{2}{b-x}\left(-f\left(\frac{b+x}{2}\right)+\frac{2}{b-x} \int_{\frac{b+x}{2}}^{b} f(u) \mathrm{d} u\right) .
\end{aligned}
$$

From (2.4)-(2.7), the identity (2.3) follows. The proof is complete.

Lemma 2.2. For $m \in(0,1]$ and $r, s>0$, we have

$$
\begin{aligned}
& H_{1}(r, s)=\int_{0}^{1} \frac{t \mathrm{~d} t}{t s^{-1}+m(1-t) r^{-1}} \\
& =\left\{\begin{array}{lr}
\frac{r s(r-m s+m s \ln (m s / r))}{(r-m s)^{2}}, & r-m s \neq 0, \\
\frac{r}{2 m}, & r-m s=0,
\end{array}\right.
\end{aligned}
$$

and

$$
\begin{aligned}
& H_{2}(r, s)=\int_{0}^{1} \frac{\mathrm{d} t}{t s^{-q}+m(1-t) r^{-q}} \\
& = \begin{cases}\frac{\ln r^{q}-\ln \left(m s^{q}\right)}{r^{q}-m s^{q}}(r s)^{q}, & r^{q}-m s^{q} \neq 0, \\
\frac{r^{q}}{m}, & r^{q}-m s^{q}=0 .\end{cases}
\end{aligned}
$$

\section{Main Results}

In this section, we will present several HermiteHadamard type inequalities for the $m-\mathrm{AH}$ convex functions.

Theorem 3.1. Let $f: \mathbb{R}_{0} \rightarrow \mathbb{R}_{+}$be differentiable, $f^{\prime} \in L([a, b])$ for $0 \leq a<b$. If $\left|f^{\prime}\right|$ is an $m-\mathrm{AH}$ convex function on $[a, b / m]$ for $m \in(0,1]$, then

$$
\begin{aligned}
& \left|\frac{1}{b-a} \int_{a}^{b} f(y) \mathrm{d} y-f(x)\right| \\
& \leq \frac{(x-a)^{2}}{4(b-a)}\left[\begin{array}{l}
2 H_{2}\left(\gamma^{1 / q}(x), \alpha(x)^{1 / q}\right) \\
-H_{1}(\gamma(x), \alpha(x))+H_{1}(u, \alpha(x))
\end{array}\right] \\
& +\frac{(b-x)^{2}}{4(b-a)}\left[\begin{array}{l}
2 H_{2}\left(\gamma^{1 / q}(x), \beta^{1 / q}(x)\right) \\
-H_{1}(\gamma(x), \beta(x))+H_{1}(v, \beta(x))
\end{array}\right],
\end{aligned}
$$


where

$$
\begin{aligned}
& u=\left|f^{\prime}(a / m)\right|, v=\left|f^{\prime}(b / m)\right|, \\
& \alpha(x)=\left|f^{\prime}\left(\frac{a+x}{2}\right)\right|, \beta(x)=\left|f^{\prime}\left(\frac{b+x}{2}\right)\right|, \\
& \gamma(x)=\left|f^{\prime}(x / m)\right| .
\end{aligned}
$$

Proof. By Lemmas 2.1 and 2.2 and the $m-\mathrm{AH}$ convexity of $\left|f^{\prime}(x)\right|$ on $[a, b / m]$, we have

$$
\begin{aligned}
& \left|\frac{1}{b-a} \int_{a}^{b} f(y) \mathrm{d} y-f(x)\right| \\
& \leq \frac{(x-a)^{2}}{4(b-a)}\left(\int_{0}^{1} t\left|f^{\prime}\left(t \frac{a+x}{2}+(1-t) a\right)\right| \mathrm{d} t\right. \\
& \left.+\int_{0}^{1}(1+t)\left|f^{\prime}\left(t x+(1-t) \frac{a+x}{2}\right)\right| \mathrm{d} t\right) \\
& +\frac{(b-x)^{2}}{4(b-a)}\left(\int_{0}^{1}(2-t)\left|f^{\prime}\left(t \frac{b+x}{2}+(1-t) x\right)\right| \mathrm{d} t\right. \\
& \left.+\int_{0}^{1}(1-t)\left|f^{\prime}\left(t b+(1-t) \frac{b+x}{2}\right)\right| \mathrm{d} t\right) \\
& \leq \frac{(x-a)^{2}}{4(b-a)}\left(\int_{0}^{1} \frac{t \mathrm{~d} t}{t\left|f^{\prime}\left(\frac{a+x}{2}\right)\right|^{-1}+m(1-t)\left|f^{\prime}(a / m)\right|^{-1}}\right. \\
& \left.+\int_{0}^{1} \frac{(1+t) \mathrm{d} t}{m t\left|f^{\prime}(x / m)\right|^{-1}+(1-t)\left|f^{\prime}\left(\frac{a+x}{2}\right)\right|^{-1}}\right) \\
& +\frac{(b-x)^{2}}{4(b-a)}\left[2 H_{2}\left(\gamma^{1 / q}(x), \beta^{1 / q}(x)\right)\right. \\
& \left.+H_{1}(\gamma(x), \beta(x))+H_{1}(v, \beta(x))\right] . \\
& +\frac{(b-x)^{2}}{4(b-a)}\left(\int _ { 0 } ^ { 1 } \frac { ( x - a ) ^ { 2 } } { t | f ^ { \prime } ( \frac { b + x } { 2 } ) | ^ { - 1 } + m ( 1 - t ) | f ^ { \prime } ( x / m ) | ^ { - 1 } } \left[2 H_{2}\left(\gamma^{1 / q}(x), \alpha(x)^{1 / q}\right)\right.\right. \\
& \left.+\int_{0}^{1} \frac{(1-t) \mathrm{d} t}{m t\left|f^{\prime}(b / m)\right|^{-1}+(1-t)\left|f^{\prime}\left(\frac{b+x}{2}\right)\right|^{-1}}\right) \\
& +(b-a)
\end{aligned}
$$

So the inequality (3.1) holds, which complete the proof. Corollary 3.1.1. Under the conditions of Theorem 3.1, (1) if $x=a$, then

$$
\begin{aligned}
& \left|\frac{1}{b-a} \int_{a}^{b} f(y) \mathrm{d} y-f(a)\right| \\
\leq & \frac{b-a}{4}\left[2 H_{2}\left(\gamma^{1 / q}(a), \beta^{1 / q}(a)\right)\right. \\
- & \left.H_{1}(\gamma(a), \beta(a))+H_{1}(v, \beta(a))\right] .
\end{aligned}
$$

(2) if $x=b$, then

$$
\begin{aligned}
& \left|\frac{1}{b-a} \int_{a}^{b} f(y) \mathrm{d} y-f(b)\right| \\
& \leq \frac{b-a}{4}\left[2 H_{2}\left(\gamma^{1 / q}(b), \alpha^{1 / q}(b)\right)\right. \\
& \left.-H_{1}(\gamma(b), \alpha(b))+H_{1}(u, \alpha(b))\right] .
\end{aligned}
$$

(3) if $x=(a+b) / 2$, then

$$
\begin{aligned}
& \left|\frac{1}{b-a} \int_{a}^{b} f(y) \mathrm{d} y-f\left(\frac{a+b}{2}\right)\right| \\
& \leq \frac{b-a}{16}\left\{\left[2 H_{2}\left(\gamma^{1 / q}\left(\frac{a+b}{2}\right), \alpha^{1 / q}\left(\frac{a+b}{2}\right)\right)\right.\right. \\
& \left.-H_{1}\left(\gamma\left(\frac{a+b}{2}\right), \alpha\left(\frac{a+b}{2}\right)\right)+H_{1}\left(u, \alpha\left(\frac{a+b}{2}\right)\right)\right] \\
& +\left[2 H_{2}\left(\gamma^{1 / q}\left(\frac{a+b}{2}\right), \beta^{1 / q}\left(\frac{a+b}{2}\right)\right)\right. \\
& \left.\left.-H_{1}\left(\gamma\left(\frac{a+b}{2}\right), \beta\left(\frac{a+b}{2}\right)\right)+H_{1}\left(v, \beta\left(\frac{a+b}{2}\right)\right)\right]\right\} .
\end{aligned}
$$

Theorem 3.2. Let $f: \mathbb{R}_{0} \rightarrow \mathbb{R}_{+}$be differentiable, $f^{\prime} \in L([a, b])$ for $0 \leq a<b$. If $\left|f^{\prime}\right|^{q}$ is an $m-\mathrm{AH}$ convex function on $[a, b / m]$ for $m \in(0,1], \quad p, q>1$, and $(1 / p)+(1 / q)=1$, then

$$
\begin{aligned}
& \left|\frac{1}{b-a} \int_{a}^{b} f(y) \mathrm{d} y-f(x)\right| \\
& \leq \frac{(x-a)^{2}}{2^{2+1 / p}(b-a)}\left[3 ^ { 1 / p } \left(2 H_{2}(\gamma(x), \alpha(x))\right.\right. \\
& \left.\left.-H_{1}\left(\gamma^{q}(x), \alpha^{q}(x)\right)\right)^{1 / q}+H_{1}^{1 / q}\left(u^{q}, \alpha^{q}(x)\right)\right] \\
& +\frac{(b-x)^{2}}{2^{2+1 / p}(b-a)}\left[3 ^ { 1 / p } \left(2 H_{2}(\gamma(x), \beta(x))\right.\right. \\
& \left.\left.-H_{1}\left(\gamma^{q}(x), \beta^{q}(x)\right)\right)^{1 / q}+H_{1}^{1 / q}\left(v^{q}, \beta^{q}(x)\right)\right] .
\end{aligned}
$$

where $u, v, \alpha(x), \beta(x), \gamma(x)$ are defined in (3.2).

Proof. By Lemma 2.1 and 2.2, the $m$-AH convexity of $\left|f^{\prime}(x)\right|^{q}$ on $[a, b / m]$, and Hölder's inequality, we have

$$
\begin{aligned}
& \left|\frac{1}{b-a} \int_{a}^{b} f(y) \mathrm{d} y-f(x)\right| \\
& \leq \frac{(x-a)^{2}}{4(b-a)}\left(\int_{0}^{1} t\left|f^{\prime}\left(t \frac{a+x}{2}+(1-t) a\right)\right| \mathrm{d} t\right. \\
& \left.+\int_{0}^{1}(1+t)\left|f^{\prime}\left(t x+(1-t) \frac{a+x}{2}\right)\right| \mathrm{d} t\right) \\
& +\frac{(b-x)^{2}}{4(b-a)}\left(\int_{0}^{1}(2-t)\left|f^{\prime}\left(t \frac{b+x}{2}+(1-t) x\right)\right| \mathrm{d} t\right. \\
& \left.+\int_{0}^{1}(1-t)\left|f^{\prime}\left(t b+(1-t) \frac{b+x}{2}\right)\right| \mathrm{d} t\right) \\
& \leq \frac{(x-a)^{2}}{4(b-a)}\left[\left(\int_{0}^{1} t \mathrm{~d} t\right)^{1 / p}\right. \\
& \times\left(\int_{0}^{1} t\left|f^{\prime}\left(t \frac{a+x}{2}+m(1-t) a / m\right)\right|^{q} \mathrm{~d} t\right)^{1 / q}+\left(\int_{0}^{1}(1+t) \mathrm{d} t\right)^{1 / p} \\
& \left.\times\left(\int_{0}^{1}(1+t)\left|f^{\prime}\left(m t x / m+(1-t) \frac{a+x}{2}\right)\right|^{q} \mathrm{~d} t\right)^{1 / q}\right] \\
& +\frac{(b-x)^{2}}{4(b-a)}\left[\left(\int_{0}^{1}(2-t) \mathrm{d} t\right)^{1 / p}\right. \\
& \times\left(\int_{0}^{1}(2-t)\left|f^{\prime}\left(t \frac{b+x}{2}+m(1-t) x / m\right)\right|^{q} \mathrm{~d} t\right)^{1 / q} \\
& +\left(\int_{0}^{1}(1-t) \mathrm{d} t\right)^{1 / p} \\
& \left.\left.+\left.f^{\prime}\left(m t b / m+(1-t) \frac{b+x}{2}\right)\right|^{q} \mathrm{~d} t\right)^{1 / q}\right]
\end{aligned}
$$




$$
\begin{aligned}
& \leq \frac{(x-a)^{2}}{2^{2+1 / p}(b-a)} \\
& \times\left[\left(\int_{0}^{1} \frac{t \mathrm{~d} t}{t\left|f^{\prime}\left(\frac{a+x}{2}\right)\right|^{-q}+m(1-t)\left|f^{\prime}(a / m)\right|^{-q}}\right)^{1 / q}\right. \\
& \left.+3^{1 / p}\left(\int_{0}^{1} \frac{(1+t) \mathrm{d} t}{m t\left|f^{\prime}(x / m)\right|^{-q}+(1-t)\left|f^{\prime}\left(\frac{a+x}{2}\right)\right|^{-q}}\right)^{1 / q}\right] \\
& +\frac{(b-x)^{2}}{2^{2+1 / p}(b-a)} \\
& \times\left[3^{1 / p}\left(\int_{0}^{1} \frac{(2-t) \mathrm{d} t}{t\left|f^{\prime}\left(\frac{b+x}{2}\right)\right|^{-q}+m(1-t)\left|f^{\prime}(x / m)\right|^{-q}}\right)^{1 / q}\right. \\
& \left.+\left(\int_{0}^{1} \frac{(1-t) \mathrm{d} t}{m t\left|f^{\prime}(b / m)\right|^{-q}+(1-t)\left|f^{\prime}\left(\frac{b+x}{2}\right)\right|^{-q}}\right)^{1 / q}\right] \\
& =\frac{(x-a)^{2}}{2^{2+1 / p}(b-a)}\left[3 ^ { 1 / p } \left(2 H_{2}(\gamma(x), \alpha(x))\right.\right. \\
& \left.\left.-H_{1}\left(\gamma^{q}(x), \alpha^{q}(x)\right)\right)^{1 / q}+H_{1}^{1 / q}\left(u^{q}, \alpha^{q}(x)\right)\right] \\
& +\frac{(b-x)^{2}}{2^{2+1 / p}(b-a)}\left[3 ^ { 1 / p } \left(2 H_{2}(\gamma(x), \beta(x))\right.\right. \\
& \left.\left.-H_{1}\left(\gamma^{q}(x), \beta^{q}(x)\right)\right)^{1 / q}+H_{1}^{1 / q}\left(v^{q}, \beta^{q}(x)\right)\right] .
\end{aligned}
$$

So the inequality (3.6) holds, which complete the proof. Corollary 3.2.1. Under the conditions of Theorem 3.2,

(1) if $x=a$, then

$$
\begin{aligned}
& \left|\frac{1}{b-a} \int_{a}^{b} f(y) \mathrm{d} y-f(a)\right| \leq \frac{b-a}{2^{2+1 / p}}\left[3^{1 / p}\right. \\
& \times\left(2 H_{2}(\gamma(a), \beta(a))-H_{1}\left(\gamma^{q}(a), \beta^{q}(a)\right)\right)^{1 / q} \\
& \left.+H_{1}^{1 / q}\left(v^{q}, \beta^{q}(a)\right)\right] .
\end{aligned}
$$

(2) if $x=b$, then

$$
\begin{aligned}
& \left|\frac{1}{b-a} \int_{a}^{b} f(y) \mathrm{d} y-f(b)\right| \leq \frac{b-a}{2^{2+1 / p}}\left[3^{1 / p}\right. \\
& \left.\times\left(2 H_{2}(\gamma(b), \alpha(b))-H_{1}\left(\gamma^{q}(b), \alpha^{q}(b)\right)\right)^{1 / q}+H_{1}^{1 / q}\left(u^{q}, \alpha^{q}(b)\right)\right] .
\end{aligned}
$$

(3) if $x=(a+b) / 2$, then

$$
\begin{aligned}
& \left|\frac{1}{b-a} \int_{a}^{b} f(y) \mathrm{d} y-f\left(\frac{a+b}{2}\right)\right| \\
& \leq \frac{b-a}{2^{4+1 / p}}\left[3 ^ { 1 / p } \left(2 H_{2}\left(\gamma\left(\frac{a+b}{2}\right), \alpha\left(\frac{a+b}{2}\right)\right)\right.\right. \\
& \left.\left.-H_{1}\left(\gamma^{q}\left(\frac{a+b}{2}\right), \alpha^{q}\left(\frac{a+b}{2}\right)\right)\right)^{1 / q}+H_{1}^{1 / q}\left(u^{q}, \alpha^{q}\left(\frac{a+b}{2}\right)\right)\right] \\
& +\frac{b-a}{2^{4+1 / p}}\left[3 ^ { 1 / p } \left(2 H_{2}\left(\gamma\left(\frac{a+b}{2}\right), \beta\left(\frac{a+b}{2}\right)\right)\right.\right. \\
& \left.\left.-H_{1}\left(\gamma^{q}\left(\frac{a+b}{2}\right), \beta^{q}\left(\frac{a+b}{2}\right)\right)\right)^{1 / q}+H_{1}^{1 / q}\left(v^{q}, \beta^{q}\left(\frac{a+b}{2}\right)\right)\right] .
\end{aligned}
$$

Theorem 3.3. Let $f: \mathbb{R}_{0} \rightarrow \mathbb{R}_{+}$be differentiable, $f^{\prime} \in L([a, b])$ for $0 \leq a<b$. If $\left|f^{\prime}\right|^{q}$ is an $m-\mathrm{AH}$ convex function on $[a, b / m]$ for $m \in(0,1], p, q>1$, and $(1 / p)+(1 / q)=1$, then

$$
\begin{aligned}
& \quad\left|\frac{1}{b-a} \int_{a}^{b} f(y) \mathrm{d} y-f(x)\right| \leq \frac{(a-x)^{2}}{4(p+1)^{1 / p}(b-a)} \\
& \times\left[\left(2^{p+1}-1\right)^{1 / p} H_{2}^{1 / q}(\gamma(x), \alpha(x))+H_{2}^{1 / q}(u, \alpha(x))\right] \\
& +\frac{(b-x)^{2}}{4(p+1)^{1 / p}(b-a)} \\
& \times\left[\left(2^{p+1}-1\right)^{1 / p} H_{2}^{1 / q}(\gamma(x), \beta(x))+H_{2}^{1 / q}(v, \beta(x))\right] .
\end{aligned}
$$

Proof. By Lemma 2.1 and 2.2, the $m$-AH convexity of $\left|f^{\prime}(x)\right|^{q}$ on $[0, b / m]$, and Hölder's inequality, we have

$$
\begin{aligned}
& \quad\left|\frac{1}{b-a} \int_{a}^{b} f(\mu) \mathrm{d} \mu-f(x)\right| \\
& \leq \frac{(x-a)^{2}}{4(b-a)}\left(\int_{0}^{1} t\left|f^{\prime}\left(t \frac{a+x}{2}+(1-t) a\right)\right| \mathrm{d} t\right. \\
& \left.+\int_{0}^{1}(1+t)\left|f^{\prime}\left(t x+(1-t) \frac{a+x}{2}\right)\right| \mathrm{d} t\right) \\
& +\frac{(b-x)^{2}}{4(b-a)}\left(\int_{0}^{1}(2-t)\left|f^{\prime}\left(t \frac{b+x}{2}+(1-t) x\right)\right| \mathrm{d} t\right. \\
& \left.+\int_{0}^{1}(1-t)\left|f^{\prime}\left(t b+(1-t) \frac{b+x}{2}\right)\right| \mathrm{d} t\right) \\
& \leq \frac{(x-a)^{2}}{4(b-a)}\left(\left(\int_{0}^{1} t^{p} \mathrm{~d} t\right)\right.
\end{aligned}
$$$$
\times\left(\int_{0}^{1}\left|f^{\prime}\left[t \frac{a+x}{2}+m(1-t) a / m\right]\right|^{q} \mathrm{~d} t\right)^{1 / q}+\left(\int_{0}^{1}(1+t)^{p} \mathrm{~d} t\right)^{1 / p}
$$$$
\left.\times\left(\int_{0}^{1}\left|f^{\prime}\left[m t x / m+(1-t) \frac{a+x}{2}\right]\right|^{q} \mathrm{~d} t\right)^{1 / q}\right)+\frac{(b-x)^{2}}{4(b-a)}\left(\left(\int_{0}^{1}(2-t)^{p} \mathrm{~d} t\right)^{1 / p}\right.
$$$$
\times\left(\int_{0}^{1}\left|f^{\prime}\left[t \frac{b+x}{2}+m(1-t) x / m\right]\right|^{q} \mathrm{~d} t\right)^{1 / q}+\left(\int_{0}^{1}(1-t)^{p} \mathrm{~d} t\right)^{1 / p}
$$$$
\left.\times\left(\int_{0}^{1} \mid f^{\prime}\left[m t b / m+(1-t) \frac{b+x}{2}\right]^{q} \mathrm{~d} t\right)^{1 / q}\right)
$$$$
\leq \frac{(a-x)^{2}}{4(p+1)^{1 / p}(b-a)}
$$$$
\times\left[\left(\int_{0}^{1} \frac{\mathrm{d} t}{t\left|f^{\prime}\left(\frac{a+x}{2}\right)\right|^{-q}+m(1-t)\left|f^{\prime}(a / m)\right|^{-q}}\right)^{1 / q}\right.
$$$$
+\left(2^{p+1}-1\right)^{1 / p}
$$$$
\left.\times\left(\int_{0}^{1} \frac{\mathrm{d} t}{m t\left|f^{\prime}(x / m)\right|^{-q}+(1-t)\left|f^{\prime}\left(\frac{a+x}{2}\right)\right|^{-q}}\right)^{1 / q}\right]
$$$$
+\frac{(b-x)^{2}}{4(p+1)^{1 / p}(b-a)}\left[\left(2^{p+1}-1\right)^{1 / p}\right.
$$$$
\left.+\left(\int_{0}^{1} \frac{\mathrm{d} t}{m t\left|f^{\prime}(b / m)\right|^{-q}+(1-t)\left|f^{\prime}\left(\frac{b+x}{2}\right)\right|^{-q}}\right)^{1 / q}\right]
$$ 


$$
\begin{aligned}
& =\frac{(a-x)^{2}}{4(p+1)^{1 / p}(b-a)} \\
& \times\left[\left(2^{p+1}-1\right)^{1 / p} H_{2}^{1 / q}(\gamma(x), \alpha(x))+H_{2}^{1 / q}(u, \alpha(x))\right] \\
& +\frac{(b-x)^{2}}{4(p+1)^{1 / p}(b-a)} \\
& \times\left[\left(2^{p+1}-1\right)^{1 / p} H_{2}^{1 / q}(\gamma(x), \beta(x))+H_{2}^{1 / q}(v, \beta(x))\right] .
\end{aligned}
$$

So the inequality (3.10) holds, which completes the proof.

Corollary 3.3.1. Under the conditions of Theorem 3.3, if $x=a$, then

$$
\begin{aligned}
& \left|\frac{1}{b-a} \int_{a}^{b} f(y) \mathrm{d} y-f(a)\right| \leq \frac{b-a}{4(p+1)^{1 / p}} \\
& \times\left[\left(2^{p+1}-1\right)^{1 / p} H_{2}^{1 / q}(\gamma(a), \beta(a))+H_{2}^{1 / q}(v, \beta(a))\right] .
\end{aligned}
$$

(2) if $x=b$, then

$$
\begin{aligned}
& \left|\frac{1}{b-a} \int_{a}^{b} f(y) \mathrm{d} y-f(b)\right| \leq \frac{b-a}{4(p+1)^{1 / p}} \\
& \times\left[\begin{array}{l}
\left(2^{p+1}-1\right)^{1 / p} H_{2}^{1 / q}(\gamma(b), \alpha(b)) \\
+H_{2}^{1 / q}(u, \alpha(b))
\end{array}\right] .
\end{aligned}
$$

(3) if $x=(a+b) / 2$, then

$$
\begin{aligned}
& \left|\frac{1}{b-a} \int_{a}^{b} f(y) \mathrm{d} y-f\left(\frac{a+b}{2}\right)\right| \\
& \leq \frac{b-a}{16(p+1)^{1 / p}}\left[\left(2^{p+1}-1\right)^{1 / p}\right. \\
& \left.\times H_{2}^{1 / q}\left(\beta\left(\frac{a+b}{2}\right), \alpha\left(\frac{a+b}{2}\right)\right)+H_{2}^{1 / q}\left(u, \alpha\left(\frac{a+b}{2}\right)\right)\right] \\
& +\frac{b-a}{16(p+1)^{1 / p}}\left[\left(2^{p+1}-1\right)^{1 / p}\right. \\
& \left.\times H_{2}^{1 / q}\left(\gamma\left(\frac{a+b}{2}\right), \beta\left(\frac{a+b}{2}\right)\right)+H_{2}^{1 / q}\left(v, \beta\left(\frac{a+b}{2}\right)\right)\right] .
\end{aligned}
$$

